\title{
Laser-Based Phase Contrast for Transmission Electron Microscopy
}

\author{
O. Schwartz ${ }^{1,3}$, J. J. Axelrod ${ }^{1,3}$, S. L. Campbel1 ${ }^{1,3}$, C. Turnbaugh ${ }^{1,3}$, A. Herman ${ }^{1,3}$, E. Planz ${ }^{1,3}$, R. M. \\ Glaeser $^{2,3}$ and H. Müller ${ }^{1,3^{*}}$ \\ ${ }^{1 .}$ Department of Physics, University of California, Berkeley, CA, USA. \\ 2. Department of Molecular and Cell Biology, University of California, Berkeley, CA, USA. \\ 3. Lawrence Berkeley National Laboratory, One Cyclotron Road, Berkeley, CA, USA. \\ *Corresponding author: hm@berkeley.edu
}

Laser control of free electrons [1] has been used to advance the exploration of matter on the atomic scale. For example, temporal modulation of electron waves with light [2,3] has enabled the study of transient processes with attosecond resolution [4]. By contrast, laser-based spatial shaping of the electron wave function has not yet been realized, even though it could be harnessed to probe radiationsensitive systems, such as biological macromolecules, at the standard quantum limit [5,6] and beyond $[7,8]$.

We demonstrate increased image contrast by laser control of the spatial phase profile of the electron wave function in transmission electron microscopy (TEM). We first realize an electron interferometer, using continuous-wave laser-induced retardation to coherently split the electron beam, and capture TEM images of the light wave. We then demonstrate Zernike phase contrast $[6,9]$ by using the laser beam to shift the phase of the electron wave scattered by a specimen relative to the unscattered wave $[5,10]$.

Electrons interact with light via the repulsive ponderomotive potential arising from stimulated Compton scattering. Due to the short electron-light interaction time in a micron-scale laser focus, retardation of the relativistic electrons used in TEM requires an intensity of tens of $\mathrm{GW} / \mathrm{cm}^{2}$. Such intensities have so far only been attained with pulsed lasers, but a cw laser is needed in order to work state-of-the-art, continuously operating TEM. The requisite laser intensity is generated by 4000 -fold resonant power enhancement in a near-concentric Fabry-Perot optical cavity with a mode waist of $w_{0}=13 \mu \mathrm{m}$. A laser system consisting of a fiber amplifier seeded by a low-power master laser supplies an input laser beam at a wavelength of $\lambda=1064 \mathrm{~nm}$.

The experiments are carried out with $80 \mathrm{keV}$ electrons, in a custom-modified TEM (FEI Titan) equipped with additional electron optics that magnify the diffraction pattern to an effective focal length of $f=20$ $\mathrm{mm}$. The cavity is suspended in the TEM column, with its axis orthogonal to the electron beam propagation direction and with the mode waist positioned close to the center of the magnified electron diffraction plane, as shown in Fig. 1. Zernike phase contrast is evident in a typical close-to-focus image (Fig. 1), showing the structure of the carbon film.

A high-intensity CW laser field generates Zernike phase contrast in a TEM and significantly increases the image contrast at low spatial frequencies. Such a phase plate will enable dose-efficient data collection in single-particle analysis of biological macromolecules, electron tomography of vitrified cells, and imaging of sensitive materials science specimens. The controllable phase shift in this device can also be used for holographic reconstruction of the post-specimen wave function.

Work in the immediate future will include working with $300-\mathrm{keV}$ electrons, which requires constructing 
a new cavity with lower-loss optical coatings to reach higher laser intensity. We will also study and optimize the imaging properties of the phase-contrast TEM, and apply it to structural biology.

\section{References:}

[1] E Jones et al., Laser Photonics Rev. 10 (2016), p. 214.

[2] A Feist et al., Nature 521 (2015), p. 200.

[3] M Kozák, N Schönenberger and P Hommelhoff, Phys. Rev. Lett. 120 (2018), p. 103203.

[4] Y Morimoto and P Baum, Nat. Phys. 14 (2018), p. 252.

[5] H Müller et al., New J. Phys. 12 (2010).

[6] RM Glaeser, Rev. Sci. Instrum. 84 (2013), p. 111101.

[7] P Kruit et al., Ultramicroscopy 164 (2016), p. 31.

[8] T Juffmann et al., Sci. Rep. 7 (2017), p. 1699.

[9] F Zernike, Physica 9 (1942), p. 686.

[10] O Schwartz et al., Opt. Express 25 (2017), p. 14453.
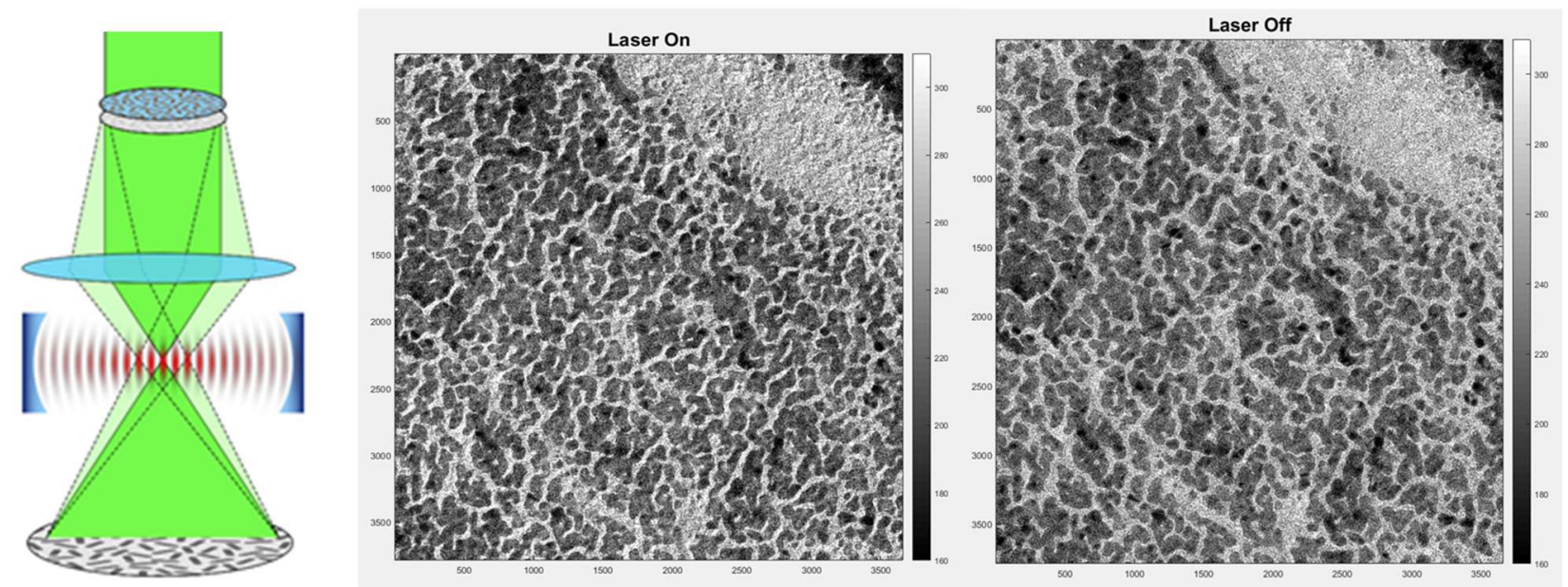

Figure 1. Left: Schematic of the experimental setup: a high-power standing laser wave, resonantly enhanced in an optical cavity, is introduced into the path of the electron beam. Not shown is a relay lens that magnifies the electron diffraction pattern. The unscattered electron beam is focused in the center of the diffraction pattern, where it passes through a single antinode of the standing laser wave. The phaseshifted unscattered beam and the scattered beam are then recombined in the image plane. Right: Phasecontrast imaging with a laser-based phase plate. A close-to-focus image of a gold-laced amorphous carbon film. Laser-based phase contrast leads to a pronounced increase of image contrast, in particular in the areas without gold that show little contrast otherwise. 